\title{
Khat Use and Its Impacts on Students' Various Aspect in Higher Educational Institutions:The Case of Mizan -Tepi University, Ethiopia
}

\author{
Brhanu Tsegay Mesele \\ Department of Sociology, Wolaita Sodo University, Ethiopia
}

\begin{abstract}
Khat chewing is one of the many maniples of drop-adjure or drug addiction. It becomes one experiential aspect of some section of society especially, the youth. Khat chewing is not uncommon among students in higher educational institutions particularly among university students. Now days, it has become a habit of significant number of students in higher educational institutions. This research is aimed at assessing impacts of Khat use among students of higher educational institutions the case of Mizan- Tepi University, Ethiopia. The research is a cross-sectional study; aimed at collecting data at one point in time and describing the study population rather than showing the patterns of change which might be witnessed over time. Key informant interviews, focus group discussions, in-depth interviews and observation used as data collection techniques. The study subjects were selected purposively/ judgmental under non-probability sampling procedure. Qualitative information recorded on notebook from FGDs, conversations with key informants, and in-depth interviews were organized and constructed coherently, analyzed descriptively- word description / sentence form. Findings of this research show that Khat use has enormous impact on student's various aspects. Although it is believed that Khat use plays a role in making students alerted and focused during long study hours, the overall result of this study shows Kaht use has negative impact on various aspects of students. The impacts can be categorized as Economic, Social, and health related, psychological impacts and impact on students' educational performance.
\end{abstract}

Khat, Khat use, impacts, educational performance

DOI: $10.7176 /$ RHSS/9-15-02

Publication date: August $31^{\text {st }} 2019$

\section{INTRODUCTION}

Khat chewing is one of the many maniples of drop-adjure or drug addiction. It becomes one experiential aspect of some section of society especially, the youth in almost every part of the glob with some exceptions. Khat chewing is not uncommon among students in higher educational institutions particularly among University students. Now days, it has become a habit of significant number of students in higher educational institutions. The use or misuse of addictive substances like Khat has become widespread among the youths especially in countries where the substance is produced and/or consumed. The percentage of khat chewing practice among regions of Ethiopia ranges from $1.1 \%$ to $53.2 \%$ with the overall prevalence of $15.3 \%$ (Tesfaye et al., 2008; Belew et al., 2000 as cited in Jemal et al. 2015:1).

Despite the fact that khat is used by 5-10 million people daily (Numan, 2012; Odenwald et al., 2010 as cited in Jemal et al. 2015:2) and its importance as cash crop among producers, there has been little interest among social scientists and other researchers regarding its effect on academic performance. In fact, there are considerable numbers of studies conducted before on the issue of origin, prevalence, trend, magnitude, khat, and khat use, and the associated effects on the user.

For instance, Tesfaye et al., 2008; Belew et al., 2000 conducted a study on how the use or misuse of addictive substances like khat is becoming increasingly widespread in recent years. A few studies have addressed the health effects of substances like cigarettes and khat in Ethiopia, focusing on psychiatric morbidity and psycho-social problems and their association with unsafe sexual behavior (Tesfaye et al., 2008; Kebede, 2002). Regarding its impact, (Beckerleg, 2010) and some other concluded that khat is an addictive substance with possible negative consequences. They believe that khat use makes users careless, less accountable and irresponsible, thereby increasing social crime. In addition khat takes a significant share of the daily expenditure of regular users and hence diverts money away from productive activities. Hence, they favor strict government control on the production and distribution of the crop (Branko, 2008).

Similarly, (Gelaw and Haile-Amlak, 2004; Mossie, 2002) concluded that there has been a dramatic rise in khat consumption recently and that its use among students especially during examination times is growing at an alarming rate. Similarly, very few studies attempted to analyze the socio- economic and health related impact of khat consumption.

\subsection{Statement of The Problem}

Khat use is becoming one part of experiential aspect of the youth in many educational institutions. In Ethiopia, It 
is true that many researchers have tried to investigate the origin of Khat, economic importance where it grows, purposes of khat use, and impacts of khat chewing. Some studies attempted to assess historical trends, determinant factors associated with Khat use, and its contributions to the national economy across time.

Some studies confirm that the adverse effects of khat use are dose dependent (Alem and Shibre, 1997). With regard to the socio-economic impact of khat use, some argue that regular use of khat has adverse effects. Loss of work hours, lower production, malnutrition, and misuse of money are some of the effects documented in the literature. This is indirectly linked to absenteeism and unemployment that in turn leads to reduction in overall economic activity.

Studies by (Baliant et al. 1994 as cited in Daniel, 2013:24) revealed that Khat induces many physical effects such as; alertness, euphoria, hyperactivity, irritability, aggressiveness, agitation, paranoid trends and tremor, and hallucination. Cox (2003), has gone further to summarize the physical effects of chewing Khat which include my driasis, dry mouth, tachycardia, arrhythmias, weight loss, and convulsions. Studies by Hassan et al. (2000) further revealed that, Khat induces a significant rise of arterial blood pressure

Nonetheless, studies on prevalence and extent of khat use among students and its likely impact on academic performance have been quite limited. Besides, their analysis lacked comprehensiveness and was shallow. Not only are such studies limited in number, but they are also limited in terms of their area of focus and the methodologies they employed.

Hence, this study is therefore, an attempt to bridge such gap regarding khat use and its impacts with specific emphasis on identifying the actual and potential impact on students' academic performance with particular reference to undergraduate students at Mizan-Tepi University, Ethiopia. In addition, the study also examined impacts of khat use on economic, social, psychological, and health aspect of students.

\section{REVIEW OF RELATED LITERATURE}

\subsection{Khat and Khat use}

The use or misuse of addictive substances like khat (Catha edulis Forsk) is becoming increasingly widespread in recent years especially among the youth in general and university students in particular (Tesfaye et al., 2008; Belew et al., 2000). As to some authors, Khat is a substance categorized with the broader class of psych stimulants like caffeine, cocoa leaves, cocaine and other substances used for their stimulating properties such as increased movement, *Corresponding author. E-mail: kimc@yonsei.ac.kr.।. Many argue that the use of Khat is both a social and a cultural activity (Al-Mutarreb et al., 2010; Manghi et al., 2009). As to (Gebissa, 2010), It is widely believed khat uses to enhance social interaction, playing a role in ceremonies such as weddings.

\subsection{Khat use and its impacts}

In their review of the effects of psych stimulants, Favrod-Coune and Broers (2010) indicate that khat use is a socially accepted habit prevalent in some Asian and African countries, covering a spectrum of beneficial, nonproblematic, problematic and addictive use. However, the authors add that data on the prevalence of and criteria for such levels of use are largely lacking (Favrod-Coune and Broers, 2010).

Other authors have documented the addictive power, tolerance and withdrawal effect of khat use (Alem and Shibre, 1997; Kalix, 1988; Kalix and Khan, 1984). With regard to psychological effects of khat use, it is widely considered in the literature that users report their subjective experiences in a positive way when consuming small amounts. They describe a feeling of well-being, a sense of exhilaration, excitement, increased energy levels, increased alertness, increased ability to concentrate, and improvement in self-esteem.

Experiences like enhanced imaginative ability and capacity to associate ideas, improvements in the ability to communicate and subjective improvements in work performance are also documented in the literature. After chewing ceases, however, unpleasant after-effects tend to dominate these experiences including sleeplessness, lack of sensation, lack of concentration and low mood. Some chewers also experience these unpleasant effects even during the chewing process, describing anxiety, tension, restlessness and hallucinations (Cox and Rampes, 2003). Moreover, it is reported that habitual khat chewing has led to decreased productivity in Ethiopia, Somalia, Uganda and Kenya.

Quite on the contrary, others argue that moderate khat use improves performance and increases work output, owing to the stimulant and fatigue postponing effects. Consequently, working hours and possibly productivity can decrease when khat is not used, because of reduced motivation (Giannini and Castellani, 1986). In relation to expenditure, khat takes significant share of regular users' budget. Kalix and Khan (1984), for instance, estimated that about $30 \%$ of all wages were spent on khat in Djibouti. There are cases where regular users secure their daily consumption of khat at the expense of vital needs, indicating dependence. Moreover, family life is damaged because of neglect, dissipation of family income and inappropriate behavior. Khat use is quoted as a factor of one in two divorces in Djibouti (Elmi, 1983).

Khat use is also considered as a source of social evils such as criminal behavior and even prostitution that are linked with attempt of acquiring funds to finance khat expenditure (Elmi, 1983). Self-harm and suicides have 
also been reported, although these incidences are rare in the literature. Each has been documented during both chewing and the subsequent intoxication phase. Suicide has been described by several authors in the context of a 'withdrawal state'. Violent acts, including homicide, are also documented usually in the context of unreasonable elusions (Alem et al., 1999; Alem and Shibre, 1997).

On the positive side, some studies have identified benefits of khat (both economic and social). Studies have shown that khat has become one of the major sources of export earnings to the national economy, a source of tax revenue for the federal and state governments, and significant cash incomes for farmers and all those involved in the khat value chain among others (Gebissa, 2008; Gebissa, 2004). In particular, the fact that khat is an evergreen and frequently harvested (up to three times a year) cash crop serving as a major source of income for the vast majority of smallholder farmers in khat growing areas becomes worth noting in this regard.

A few studies have also tried to indicate the lack of definitive scientific evidence on the harm associated with khat use as this would ruin the livelihood of many dependent on this economically significant crop in one way or another, particularly in the absence of appropriate alternative policy (Gebissa, 2010, Klein and Metaal, 2010, Gebissa, 2008). Other studies have largely focused on the determinants of khat use. Determinants of khat use and its nature as a commodity may vary from place to place even in areas where its consumption is quite common. For instance, using household surveys from Yemen and Djibouti, Branko (2008) analyzed determinants of khat consumptions. The results confirm huge importance of khat in daily life; with about onehalf in Djibouti and 70\% in Yemen of all households reporting at least one user.

But in Yemen, khat consumption is astonishingly flat across income groups, age, and between rural and urban areas. In Yemen, khat was found to be a normal good and there is no indication that its use substitutes for food. In Djibouti, however, khat consumption increases with income, and seems to be a substitute for food consumption. In both countries, nevertheless, there is a strong gender bias in the use, where men are much more likely to use khat than women. With reference to the Ethiopian case, empirical studies point out that there has been a dramatic rise in khat consumption recently and that its use among students especially during examination times is growing at an alarming rate (Gelaw and Haile-Amlak, 2004; Mossie, 2002).

However, by a study done in Saudi Arabia Khat chewers were found to have had poorer academic performance compared to no chewers. Another study done on students of Jimma University in 2002 also reported that non chewers had statistically significant higher cumulative grade point average compared to Khat chewers. In this study, large proportions of the students reported that they used alcohol and cigarette with and/or after Khat chewing. Alcohol is commonly employed as a means to help decrease or eliminate the exciting/stimulating effects of Khat chewing. Simultaneous use of cigarette and other psychoactive substances with Khat has also been reported by other studies. This shows that Khat chewing has a far reaching implication in regard to leading to the use of other addictive substances which could result in a multiple addiction with dangerous consequences. Various proportions of the chewers recounted they experienced problems such as loss of sleep, loss of appetite, constipation, and loss of weight as the main health problems. Negative consequences of Khat chewing, as well as its perceived effects, on health have been reported by different reports.

The adverse effect of khat on reproductive health includes genotoxic and teratogenic effects on the fetus, causing low birth weight. Moreover, khat also affects the potency of male sexuality by affecting spermatogenesis and plasma testosterone concentration. Growing evidence shows that khat use is one of the risk factors for taking risky sexual behaviors which fuel the widespread effect of sexually transmitted infections (STIs)especially Human Immunodefciency Virus (HIV) infection1. Besides khats' effect on mental, physical, and reproductive health, it is one of the factors causing socio-economic problems. These include; social isolation, family breakdown, neglect of social responsibilities and spending more than half of the domestic budget on khat (African Health Sciences Vol. 17 Issue 1, March, 2017 175). Moreover, khat is also reported as a risk factor for poor working memory, poor academic performance, reduced productivity and increased daily financial expenditure. Despite these harmful consequences, the cultivation and use of khat is on arise especially in Yemen, Arabian peninsula, East Africa, and Ethiopia in particular.

In Ethiopia, khat has started to rapidly replace the precious cereal, coffee, fruits and other crops found in the highlands of Ethiopia. Farmers prefer khat due to different reasons including, it's profitability as a cash crop than others, less vulnerability to drought, and less labor power cost required for its cultivation. However, khat producing farmers in Ethiopia start consuming khat and this culture is also continued expanding to the nearby secondary schools and urban dwellers. Khat or other psycho-active substance use at an early age leads to a complicated type of substance use in the remaining adulthood period of an individual. Youths' substance use, therefore, contributes to the creation of a community with; substance use dependence, juvenile delinquencies, crimes, socio-economic and other public health problems.

The Magnitude of students' khat use in Ethiopia ranges from $13.4 \%$ to $41 \%$ for lifetime and $6.3 \%-33.1 \%$ for current (past 30 days). Among the top students' expressed reasons for khat use is; to get energized for study, to stay awake or avoid sleep and to increase academic performance. Factors increasing the odds of students' khat use include; male gender, having friend chewing khat, having family members chewing khat, Muslim religion, 
pocket money, living alone during school age, cigarette smoking, financial freedom, parents' educational levels, living in rural area, and others (Jemal et al. , 2015).

Some studies confirm that the adverse effects of Khat use are dose dependent (Alem and Shibre, 1997). With regard to the socioeconomic impact of khat use, some argue that regular use of khat has adverse socioeconomic effects. Loss of work hours, lower production, malnutrition, and misuse of money are some of the effects documented in the literature. This is indirectly linked to absenteeism and unemployment that in turn leads to reduction in overall economic activity. Moreover, it is reported that habitual khat chewing has led to decreased productivity in Ethiopia, Somalia, Uganda and Kenya. Quite on the contrary, others argue that moderate khat use improves performance and increases work output, owing to the stimulant and fatigue postponing effects. Consequently, working hours and possibly productivity can decrease when khat is not used, because of reduced motivation. (Giannini and Castellani, 1986) In relation to expenditure, khat takes significant share of regular users' budget. Kalix and Khan (1984), for instance, estimated that about $30 \%$ of all wages were spent on khat in Djibouti.

On the 'positive side', some studies have identified benefits of khat (both economic and social). Studies have shown that khat has become one of the major sources of export earnings to the national economy, a source of tax revenue for the federal and state governments, and significant cash incomes for farmers and all those involved in the khat value chain among others (Gebissa, 2008; Gebissa, 2004). In particular, the fact that khat is an ever-green and frequently harvested (up to three times a year) cash crop serving as a major source of income for the vast majority of smallholder farmers in khat growing areas becomes worth noting in this regard.

A few studies have also tried to indicate the lack of definitive scientific evidence on the harm associated with khat use as this would ruin the livelihood of many dependent on this economically significant crop in one way or another, particularly in the absence of appropriate alternative policy (Gebissa, 2010, Klein and Metaal, 2010, Gebissa, 2008).

\section{METHODOLOGY}

To achieve objective of the study, qualitative study approach has been used. The research is a cross-sectional study; aimed at collecting data at one point in time and describing the study population rather than showing the patterns of change which might be witnessed over time. The study employed primary and secondary data sources. The sources of the information were key informants, focus groups, fields, and Observation. The secondary sources of information include books, articles, research journals, and different reports. Key informant interviews, focus group discussions, in-depth interviews and observation used as data collection techniques. Checklists were designed to manage the, focus group discussions, Key informant interviews, and in-depth interviews.

The study subjects were selected purposively/ judgmental under non-probability sampling procedure. This was due the difficulty of gaining full list of chat chewer students from any source. Finally, the researcher conducted three FGDs containing 8, 7, and 7 discussants in each FGD. Besides, key informant interviews were conducted with three different individuals. In-depth interviews were also made with 2 purposely selected individuals who have been engaged on Khat use relatively for long period of time. Besides, an interview was also conducted with one psychologist, Sociologist, health science professional so as to investigate information associated with the psychological, social and health related impacts. Qualitative information recorded on notebook from FGDs, conversations with key informants, and in-depth interviews were organized, constructed coherently, and analyzed thematically.

\section{STUDY RESULTS}

This section of the paper deals with various impacts of Khat use on students' different aspects. In spite of the students Khat use at different occasions, there are many adverse impacts of Khat use on different aspects of students. Impacts of Khat use investigated through qualitative method are categorized as economic, social, psychological, health related impacts and impact on educational performance of students.

Most of studies conducted in Ethiopia and other countries reported that students are using khat for its stimulant effect. Among the reported reasons by the study participants in this study; to wake up or avoid sleep, to get energized for study, and to increase academic performance are included. As limitation of all cross-sectional surveys, this study didn't intend to explain beyond determining the odds of students' poor academic performance due to khat use.

\subsection{Various of Impacts of Khat use}

Majority of study participants agreed that Khat plays a role in making students awake and stay alerted during long studying hours. It helps students stay focused on what they are doing. In addition, they believed that khat use makes individuals pleasant and social conformists. Furthermore, Khat chewing serves as a way-out from stress, anxiety and personal disturbances. However, this needs further investigation by professional researchers interested in the area. Despite the perceived role of Khat use as stated above, in making students awake and stay 
alerted during long studying hours, on the contrary, there are also other adverse impacts of Khat use on students various aspects.

\section{a) Economic Impact}

Although there might be variations in the amount of money students receive from their parents, most students are dependent on their parents or families. Students demand their families to send them some money for different purposes. This might include, for shopping clothes, shoes, stationary materials such as, material photocopy and printouts. However, there are cases where regular users secure their daily consumption of khat at the expense of vital needs, indicating dependence. Students who use Khat tend to spend the cash they receive from parents to buy Khat to satisfy the usual intake of their habit. In such cases, students ask their parents to send additional cash frequently than the usual bases.

Moreover, due to using of money for other than the intended purpose, students ' faced shortage of money to fulfill their basic needs during their stay in the academic institutions. Ultimately, this uneconomic culture of students has also an impact on their families' economy which was consistent with other findings like (Elmi, 1983) noted that family life is damaged because of neglect, dissipation of family income and inappropriate behavior.

\section{b) Social Impacts}

Society's attitude towards khat use may vary from one area to another due to different factors. For instance, there are cases in which khat use is culturally accepted behavior in some societies while considered as against societal vales and norms in other societies. Students who came from areas that condemned khat use for different reason might face isolation and detachment from their friends who are from the same place of origin. In such circumstances, social acceptance and integration of students who use khat can be declined. Most of the Study participants added that the increasingly widespread of khat chewing habit increases development of unhealthy risky behaviors among students. In addition, findings of this study indicated that there is a significant association of khat use with cigarette smoking, and consumption of coffee, and alcohol beverages. In the long run, this led to drug dependence, and addiction which are acute social problems of the youth. This finding is in line with (Jemal et al 2015) study results which were conducted in Mana district, Jimma zone, southwestern Ethiopia.

\section{c) Psychological Impact}

Besides making students vulnerable to some psychological diseases such as, schizophrenia, stress, sleeping disorder, hallucination, depression, anxiety, psychosis, habitual khat use pushes students to develop a positive attitude towards Khat use. This emanates from an assumption that 'nothing can help to escape from stress and mental disturbances except khat use.' Afterwards, students develop a distorted belief and attitude towards different kinds of therapies which are helpful to revive from different psychic disorders.

\section{d) Health related Impact}

Regular khat users tend to be alcoholics and smokers. This by itself has an impact on different organs of the body such as on organs of the respiratory system. For some students, khat use affects their appetite. They become less interested to take meals and insufficient food intake can resulted in malnutrition and reduction of body`s immunity capacity. This finding is almost consistent with Zeleke et al. report who finds out that perceived health associated impacts of khat use such as sleeping disorder, tooth staining, and loss of appetite constipation, gastritis, and hypertension. The most common mode of Khat consumption demands consistent chewing, a situation which strains the cheek and results to periodontal infections, mouth dryness dental carries and pains in the mouth cavity (Danowski, 1996). According to Kennedy (1987), Khat consumption cause lesions to the supporting structures of the teeth namely gingivitis and also results to tooth mortality. Chewing of Khat in males temporarily interferes with urine flow

\section{e) Impact on Educational performance}

Study participants agreed that Khat plays a role in making students awake and stays alerted during long studying hours. It helps students stay focused on what they are doing. However, results of this study indicated that those khat chewing students in this study had a poor chance of performing well in their academics or in another way had a likelihood of failing in their academics as a result of khat use which is in accordance with other study findings. As to the findings of this study, Khat chewing sessions are time consuming let students stay out of library, reduce study hours and time shall be used for independent learning and doing assignments. This was also proven by the researcher in which students spent three-five hours on average in a day.

In summary, khat use has negative impact on academic performance. However, among students who are users, it's the heavy users that are affected the most, while light users were relatively better performers depending on the study results from FGDs, key informant interviews and in-depth interview conducted in this study. This is in line with previous findings that the impact of khat is dose dependent especially in the fields of medical health and pharmacology (Nyachieo et al., 2012; Nyongesa et al., 2008 as cited in Jemal et al. 2015:1).

\section{CONCLUSION AND RECOMMENDATIONS 5.1 Conclusion}

In this paper, the researcher tried to examine the impacts of Kaht use on various aspects of students. Impacts of 
Khat use can be categorized as economic, social, psychological, health related impacts and impact on educational performance of students. As to some of study participants, Khat plays a role in making students awake and stays alerted during long studying hours. It helps students stay focused on what they are doing. In addition, they believed that khat use makes individuals pleasant and social conformists. Furthermore, Khat chewing serves as a way-out from stress, anxiety and personal disturbances. However, this needs further investigation by professional researchers interested in the area.

Despite the perceived 'positive role' that Khat use plays, there are also other adverse impacts of Khat use on students various aspects. Students demand their families to send them some money for different purposes. However, there are cases where regular Khat users secure their daily consumption of khat at the expense of vital needs, indicating dependence. This uneconomic culture of students has also an impact on their families' economy which was consistent with some other findings.

Students who came from areas that condemned khat use for different reason might face isolation and detachment from their friends who are from the same place of origin. In such circumstances, social acceptance and integration of students who use khat can be declined. Most of the Study participants added that the increasingly widespread of khat chewing habit increases development of unhealthy risky behaviors among students. In the long run, this led to drug dependence, and addiction which are acute social problems of the youth. Besides, makes students vulnerable to some psychological diseases and habitual khat use pushes students to develop a positive attitude towards Khat use.

Khat chewing sessions are time consuming let students out of library, reduce study hours and time shall be used for independent learning and doing assignments. This was also proven by the researcher in which students spent three-five hours on average in a day.

\subsection{Recommendations}

Considering the multidimensional impacts of Khat use, the researcher needs to recommend the following points. First, there should be awareness rising among students about the negative consequences of khat use on students' various aspects including an impact on academic performance. This can be organized and held by different clubs established in university compounds to enhance students' awareness with regard to the impacts of Khat use. Second, there has to be a concerted effort to improve the presence and quality of various recreational facilities in academic institutions for which such facilities to enable students spent their leisure time. This is due to that absence of such facilities would be considered as one of the pathways headed students to Khat use. Furthermore, this would have an important implication to gain an understanding of the possible factors contributing for students' khat use and act accordingly.

\section{REFERENCES}

Al-Mutarreb A, Al-Habori Mand Broadley KJ (2010). Khat chewing, cardiovascular diseases and other internal medical problems: the current situation and directions for future research. J. Ethnopharmacol.132 (3)

Alem A, Shibre T (1997). Khat induced psychosis and its medico-legal implication: a case report. Ethiopian Med. J. 35(2)

Beckerleg S (2010). East African discourses on khat and sex. J. Ethnopharmacol 132(3)

Belew M, Kebede D, Kassaye M, Enquoselassie F (2000). The magnitude of khat use and its association with health, nutrition and socioeconomic status. Ethiopian Med. J. 38(1)

Branko M (2008). Qat expenditures in Yemen and Djibouti: an empirical analysis. J. Afr. Econ. 17(5).

Cox G, Rampes H (2003). Adverse effects of khat: a review. Adv. Psychiatric Treatment 9(6)

Daniel J. N. Ngeranwa, (BEd. Sci.) Impact of Khat Cultivation On Educational Performance Among Upper Primary Schools Pupils In Gachoka Division, Embu County, Kenya

Elmi AS (1983). The chewing of khat in Somalia. J. Ethnopharmacol. 8(2)

Favrod-Coune T, Broers B (2010). The health effect of psychostimulants: a literature review. Pharmaceuticals $3(7)$

Gebissa E (2004). Leaf of Allah: khat and agricultural transformation in Harerge, Ethiopia, Ohio State University Press.

Gebissa E (2008). Scourge of life or an economic lifeline? Public discourses on khat (Catha edulis) in Ethiopia. Substance Use and Misuse 43(6)

Gebissa E (2010). Khat in the Horn of Africa: Historical perspectives and current trends. J. Ethnopharmacol. $132(3)$

Gebre-Egziabher T (2010). Poverty and Poverty Reduction in Ethiopia. J. Poverty Alleviation Int. Dev. 1(1): 2154.

Gelaw Y, Haile-Amlak A (2004). Khat chewing and its socio-demographic correlates among staff of Jimma University. Ethiopian J. Health Dev. 18(3)

Giannini AJ, Castellani S (1982) A manic-like psychosis due to khat Catha Edulis Forsk. Clin. Toxicol. 19(5) 
Jemal Abafita, Badassa Wolteji Chala, Kasahun Eba, Kyung-Ryang Kim, and Chang Soo Kim (2015). Khat use and its impact on academic performance: The case of Jimma University, Ethiopia

Kalix P (1988). Khat: a plant with amphetamine effects. J. Substance Abuse Treatment 5(3)

Kalix P, Khan I (1984). Khat: an amphetamine-like plant material. Bull. World Health Organ. 62(5):681-686.

Kebede Y (2002). Cigarette smoking and khat chewing among college students in North West Ethiopia. Ethiopian J. Health Dev. 16(1)

Kennedy JG, Teague J, Rokaw W, Cooney E (1983). A medical evaluation of the use of Khat in North Yemen. Soc. Sci. Med. 17(12)

Mossie A (2002). The prevalence and socio-demographic characteristics of khat chewing in Jimma town, South Western Ethiopia. Ethiopian J. Health Sci. 12(2)

Tesfaye F, Byass P, Berhane Y, Bonita R, Wall S (2008). Association of smoking and khat (Catha Edulis Forsk) use with high blood pressure among Adults in Addis Ababa, Ethiopia, 2006. Prevent. Chronic Dis. 5(3)

Zeleke A, Awoke W, Gebeyehu E, Ambaw F. Khat chewing practice and its Perceived health effects among communities of Dera Woreda, Amhara region, Ethiopia. Open J Epidemiol 2013;3:160-8 